\title{
Reversible Isolated Accessory Nerve Palsy due to a Large Thrombosed Vertebral Aneurysm
}

\author{
Hisayasu Saito $^{a}$ Satoshi Kuroda ${ }^{a, b}$ Shunsuke Terasaka ${ }^{a}$ \\ Takeshi Asano $^{a}$ Naoki Nakayama ${ }^{a}$ Kiyohiro Houkin ${ }^{a}$ \\ ${ }^{a}$ Department of Neurosurgery, Hokkaido University Graduate School of Medicine, \\ Sapporo, and ${ }^{b}$ Department of Neurosurgery, Graduate School of Medicine and \\ Pharmaceutical Science for Education, University of Toyama, Toyama, Japan
}

\section{Key Words}

Accessory nerve palsy · Intracranial aneurysm · Thrombosed aneurysm · Vertebral artery

\begin{abstract}
Objective: Isolated accessory nerve palsy due to intracranial disorders is uncommon because intracranial accessory nerve injury usually occurs in case of a skull base tumor or trauma, resulting in one of multiple cranial nerve palsies. We report a very rare case of isolated accessory nerve palsy due to a large thrombosed aneurysm of the intracranial vertebral artery. Full recovery was achieved after surgery. Case Report: A patient complaining of transient numbness in the right side was referred to our hospital. An MRI indicated a large thrombosed aneurysm of the right vertebral artery. The aneurysm severely compressed the medulla oblongata. First, the proximal vertebral artery (VA) was clipped with an aneurysm clip to reduce the pressure inside the aneurysm. However, cerebral angiography revealed a partial recanalization of the right VA. The patient then underwent coil embolization of the right VA just proximal to the aneurysm clip. Subsequently, the right VA was completely obliterated. The patient was discharged without any neurological deficit. Two weeks later, however, she complained of right shoulder pain. Physical and neurological examinations demonstrated atrophy of the right trapezius and sternocleidomastoid muscle, leading to a deepening of the right supraclavicular fossa. The symptoms were considered to result from the right isolated accessory nerve palsy. Follow-up MRI showed that the VA aneurysm gradually decreased in size over a period of several months. At the same time, her symptoms disappeared completely. Conclusion: We should keep in mind that isolated accessory nerve palsy can be caused by a large or giant vertebral aneurysm.

(c) 2013 S. Karger AG, Basel
\end{abstract}


Saito et al.: Reversible Isolated Accessory Nerve Palsy due to a Large Thrombosed Vertebral Aneurysm

\section{Introduction}

Isolated accessory nerve palsies are most commonly reported as one of the complications after surgery involving the posterior cervical triangle. Numerous alternative etiologies, such as traction, acute brachial neuritis, blunt and penetrating trauma and internal jugular vein cannulation, are also described $[1,2]$. In most of these cases, injury of the extracranial spinal accessory nerve causes its isolated palsy. On the other hand, isolated accessory nerve palsy due to intracranial disorders is uncommon because intracranial accessory nerve injury usually occurs in case of a skull base tumor or trauma, resulting in one of multiple cranial nerve palsies [3]. Here, we report a very rare case of isolated accessory nerve palsy due to a large thrombosed aneurysm of the intracranial vertebral artery. Full recovery was achieved after surgery.

\section{Case Report}

A 70-year-old woman complaining of transient numbness in the upper right and lower limbs was referred to our hospital. Physical and neurological examinations revealed no definite abnormalities. However, MRI indicated a large mass lesion in the posterior fossa. The maximal diameter was $22 \mathrm{~mm}$. The mass severely compressed the medulla oblongata and caused a high signal intensity lesion, suggesting a vasogenic edema. Both T1- and T2weighted MRI showed a flow void signal and mixed signal intensity area inside the mass, suggesting a partially thrombosed vertebral aneurysm (fig. 1a). MRA disclosed a thrombosed large aneurysm of the right vertebral artery (VA) (fig. 1b).

The proximal VA was exposed through right suboccipital craniotomy and was clipped with one titanium aneurysm clip in order to reduce the pressure inside the aneurysm. The postoperative course was uneventful. No neurological deterioration occurred. Postoperative MRA showed the disappearance of the flow void signal inside the aneurysm. One month later, however, cerebral angiography revealed a partial recanalization of the right VA, probably because one titanium aneurysm clip was not enough to obliterate the sclerotic VA. She then underwent coil embolization of the right VA just proximal to the aneurysm clip. Subsequently, the right VA was completely obliterated. She was discharged without any neurological deficit. However, a couple of weeks later she complained of mild hoarseness and right shoulder pain. Physical and neurological examinations showed the atrophy of the right trapezius and sternocleidomastoid muscle, leading to the deepening of the right supraclavicular fossa (fig. 2a). No other cranial nerve deficits were observed. The symptoms were considered to result from right isolated accessory nerve palsy. Radiological examinations showed no newly developed lesion such as cerebral infarction in the brain stem. There was no significant change in size of the VA aneurysm. As a result, the isolated accessory nerve palsy was considered to be a delayed complication due to aneurysmal compression on the intracranial portion of the accessory nerve.

Follow-up MRI showed that the VA aneurysm gradually decreased in size over a period of several months. The deformity of the medulla oblongata markedly improved and its high signal intensity lesion completely disappeared (fig. 1c). At the same time, the patient's hoarseness completely resolved. The deepening of the right supraclavicular fossa also disappeared completely (fig. $2 \mathrm{~b}$ ). 
Saito et al.: Reversible Isolated Accessory Nerve Palsy due to a Large Thrombosed

\section{Discussion}

The accessory nerve is composed of the cranial and the spinal roots. The cranial root arises from the inferior portion of the nucleus ambiguous in the medulla and is designated to innervate the laryngeal muscles. The spinal root originates from the anterior horn cells of the upper five or six cervical vertebrae and enters the posterior cranial fossa via the foramen magnum where it merges with the cranial root. They then exit through the jugular foramen. The spinal accessory nerve branches off, passes under the posterior belly of the digastric muscle to supply the sternocleidomastoid muscle, finally transverses the posterior cervical triangle, superficial to the prevertebral fascia, and terminates with its branches in the deep surface of the trapezius muscle [2, 4].

As aforementioned, accessory nerve injury commonly occurs when the nerve crosses the posterior cervical triangle. Its superficial location makes the nerve extremely susceptible to injury. The most common cause of accessory nerve palsy is surgical damage during neck dissections $[1,2]$.

The typical clinical features of spinal accessory nerve palsy can be listed as atrophy/weakness of the trapezius muscle and moderate winging of the scapula and less frequently that the sternocleidomastoid muscle may be involved because branches of the spinal accessory nerve that cross the posterior cervical triangle terminate the trapezius muscle [2]. As a result, the most common complaint is the inability to raise the affected arm above the horizontal and/or shoulder droop. The patients usually complain of ipsilateral neck and shoulder pain which sometimes radiates into the arm. Since the accessory nerve is entirely a motor component, this pain was thought to be caused by the placement of tension on the shoulder joint or elements of the brachial plexus secondary to chronic depression $[2,5]$.

In our case, the patient suffered from right shoulder pain and from wasting of the right trapezius and the sternocleidomastoid muscle - this led to the deepening of the supraclavicular fossa. These were considered as symptoms of spinal root palsy. In addition, she had mild hoarseness.

These findings strongly suggest that both the cranial and the spinal roots of the accessory nerve were damaged in the intracranial region (right beside the medulla oblongata). It is unlikely that surgical interventions caused the presented symptoms because her neurological status did not deteriorate right after surgery.

It is unclear why the isolated accessory nerve palsy occurred two months after surgical treatment. Sudden cessation of intra-aneurysmal flow or progression of intra-aneurysmal thrombosis may transiently increase the pressure on the surrounding accessory nerve. However, a gradual decrease in aneurysmal size completely eliminated the symptoms. In this case, electromyography was not performed. However, it would be valuable to identify the cause of the reversible atrophy of the trapezius and sternocleidomastoid muscle in such cases even more clearly.

\section{Conclusion}

We report a rare case of reversible, isolated accessory nerve palsy due to a large thrombosed aneurysm of the VA. Neurological assessment suggests that the damage of the cranial fibers caused hoarseness, and the damage of the spinal fibers caused the deepening of the supraclavicular fossa and the shoulder pain. We should keep in mind that the above- 
mentioned symptoms can occur due to isolated accessory nerve palsy which is caused by a large or giant vertebral aneurysm.

\section{Disclosure Statement}

The authors declare no conflict of interest.

\section{References}

1 Baker SK: Isolated spinal accessory mononeuropathy associated with neurogenic muscle hypertrophy: restricted neuralgic amyotrophy or stretch-palsy? A case report. Arch Phys Med Rehabil 2008;89:559-563.

$\checkmark 2$ Ozdemir O, Kurne A, Temuçin C, Varli K: Spontaneous unilateral accessory nerve palsy: a case report and review of the literature. Clin Rheumatol 2007;9:1581-1583.

3 Lunardi P, Mastronardi L, Farah JO, De Biase C, Trasimeni G, Gualdi GF: Spinal accessory nerve palsy due to neurovascular compression. Report of a case diagnosed by magnetic resonance imaging and magnetic resonance angiography. Neurosurg Rev 1996;19:175-178.

4 Wills AJ, Sawle GV: Accessory nerve palsies. Pract Neurol 2010;10:191-194.

5 Mariani PP, Santoriello P, Maresca G: Spontaneous accessory nerve palsy. J Shoulder Elbow Surg 1998;7:545-546.
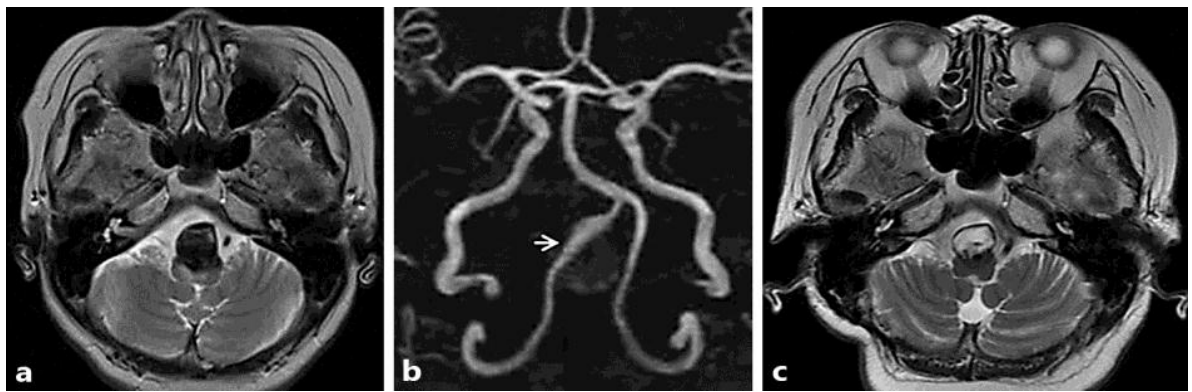

Fig. 1. Preoperative MRI indicating a partially thrombosed large aneurysm, its maximum diameter 22 $\mathrm{mm}$, in the posterior fossa. It compressed the medulla oblongata posteriorly (a). Preoperative MRA disclosing a large thrombosed aneurysm of the right VA (arrow) (b). Follow-up MRI showing that the VA aneurysm decreased in size several months after treatment (c). 


\section{Case Reports in Neurology}

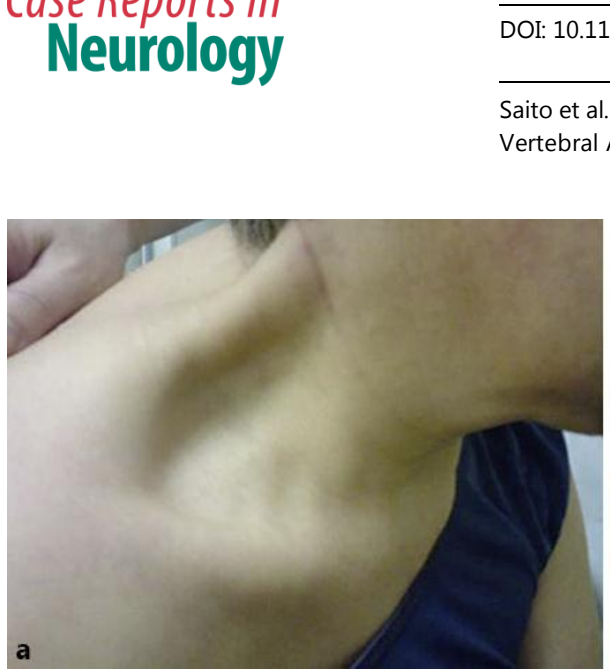

(C) 2013 S. Karger AG, Basel www.karger.com/crn

Saito et al.: Reversible Isolated Accessory Nerve Palsy due to a Large Thrombosed Vertebral Aneurysm

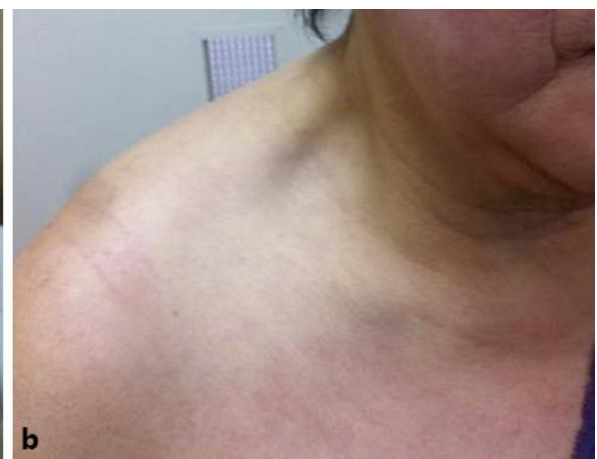

Fig. 2. A symptom of accessory nerve palsy. The right trapezius and the sternocleidomastoid muscle were wasting and these changes led to the deepening of the supraclavicular fossa (a). A gradual decrease in aneurysmal size completely eliminated the symptoms (b). 